\title{
Inner-shell excitation of open-shell atoms: a spin-dependent localized Hartree-Fock density-functional approach
}

\author{
Zhongyuan Zhou and Shih-I Chu \\ Department of Chemistry, University of Kansas, Lawrence, KS 66045, USA
}

Received 14 August 2007, in final form 15 September 2007

Published 5 November 2007

Online at stacks.iop.org/JPhysB/40/4379

\begin{abstract}
We present a spin-dependent localized Hartree-Fock (SLHF) densityfunctional approach for the treatment of inner-shell excited states of openshell atomic systems. In this approach, the electron spin-orbitals and singleSlater-determinant energies of an electronic configuration are computed by solving the Kohn-Sham (KS) equation with SLHF exchange potential. The multiplet energy of an inner-shell excited state is evaluated from the singleSlater-determinant energies in terms of Slater's diagonal sum rule. Based on this procedure, we perform calculations of the total and excitation energies of inner-shell excited states of open-shell atomic systems: $\mathrm{Li}, \mathrm{B}, \mathrm{Ne}^{+}, \mathrm{Ne}^{2+}, \mathrm{Ne}^{3+}$ and $\mathrm{Na}$. In the calculation, the electron correlation effect is taken into account via the correlation potential and energy functional of Perdew and Wang (PW) or of Lee, Yang and Parr (LYP). The calculated results are in good agreement with the available experimental and other ab initio theoretical data. In addition, new results for highly excited inner-shell states are also presented.
\end{abstract}

\section{Introduction}

Due to its computational simplicity and efficiency, density-functional theory (DFT) $[1,2]$ has been widely used as a powerful ab initio approach for the study of many-electron quantum systems in the last several decades. Conventional DFT using traditional exchange-correlation (XC) potentials obtained from uniform electron gas, such as local density approximation (LDA) [3, 4] and generalized gradient approximation (GGA) [4-6], is a ground-state approach. Because of incomplete cancellation of spurious self-interaction energies and inherent degeneracy (due to the use of spin- and angular-momentum-independent local potentials) of the $\mathrm{XC}$ potential, the differences of energy eigenvalues of unoccupied and occupied orbitals obtained from the Kohn-Sham (KS) equation [2] cannot be rigorously defined as excitation energies. However, the KS energy eigenvalues can serve as good zerothorder excited-state energies provided they are obtained by solving the KS equation with a high-quality XC potential $[7,8]$. 
Recently, an exchange (X)-only localized Hartree-Fock (LHF) density-functional theory has been proposed and successfully applied to ground-state calculations of atomic and molecular systems [7]. In this X-only DFT, the exchange potential in the KS equation is a LHF exchange potential derived under the assumption that the X-only KS determinant is equal to the Hartree-Fock (HF) determinant. The LHF potential is self-interaction free and exhibits the correct long-range behaviour. It requires only the use of occupied orbitals and is dependent on the orbital symmetry of the state.

We have developed, as an extension of this theory, a spin-dependent localized HartreeFock (SLHF) density-functional approach for the excited-state calculation of atomic and molecular systems [9]. In this approach, the exchange potential in the KS equation is an exact nonvariational SLHF exchange potential obtained in a similar way as the LHF potential by assuming that for excited states the X-only KS determinant is also equal to the HF determinant [9]. The SLHF potential is an analogue of the LHF potential but for both the ground and excited states. This approach together with Slater's diagonal sum rule [10] have been successfully used to calculate the energies of multiply excited states of valence electrons of atomic systems [9] and inner-shell excited states of close-shell atomic systems [11] with accurate results.

In this paper, we extend the SLHF density-functional approach to the calculation of total and excitation energies of inner-shell excited states of open-shell atomic systems: $\mathrm{Li}, \mathrm{B}, \mathrm{Ne}^{+}$, $\mathrm{Ne}^{2+}, \mathrm{Ne}^{3+}$ and $\mathrm{Na}$. In the calculation, the electron correlation effect is taken into account through the correlation potential and energy functional of Perdew and Wang (PW) [12] or of Lee, Yang and Parr (LYP) [13]. The calculated results are in good agreement with the available theoretical and experimental data. We also present some new results for the highly excited inner-shell states for the first time.

\section{Theoretical method}

In the spin-dependent density-functional approach, a spin-orbital $\varphi_{i \sigma}(\mathbf{r})$ of the $i$ th electron with spin $\sigma$ ( $\sigma=\alpha$ and $\beta$ for spin-up and spin-down, respectively) and its orbital energy $\varepsilon_{i \sigma}$ are determined by the KS equation

$$
H_{\sigma}(\mathbf{r}) \varphi_{i \sigma}(\mathbf{r})=\varepsilon_{i \sigma} \varphi_{i \sigma}(\mathbf{r})
$$

where $H_{\sigma}(\mathbf{r})$ is the KS Hamiltonian

$$
H_{\sigma}(\mathbf{r})=-\frac{1}{2} \nabla^{2}+V_{\sigma}^{\text {eff }}(\mathbf{r})
$$

and $V_{\sigma}^{\text {eff }}(\mathbf{r})$ is the local effective potential given by

$$
V_{\sigma}^{\text {eff }}(\mathbf{r})=V_{\text {ext }}(\mathbf{r})+V_{H}(\mathbf{r})+V_{x c \sigma}(\mathbf{r}) \text {. }
$$

In equation (3), $V_{\text {ext }}(\mathbf{r})$ is the external potential, $V_{H}(\mathbf{r})$ is the Hartree potential (classical Coulomb electrostatic potential between electrons) and $V_{x c \sigma}(\mathbf{r})$ is the spin-dependent $\mathrm{XC}$ potential.

The external potential $V_{\text {ext }}(\mathbf{r})$ is known exactly for a given atomic system. The Hartree potential $V_{H}(\mathbf{r})$ is calculated from

$$
V_{H}(\mathbf{r})=\int \frac{\rho\left(\mathbf{r}^{\prime}\right)}{\left|\mathbf{r}-\mathbf{r}^{\prime}\right|} \mathrm{d} \mathbf{r}^{\prime},
$$

where $\rho(\mathbf{r})=\rho_{\alpha}(\mathbf{r})+\rho_{\beta}(\mathbf{r})$ is the total electron density and $\rho_{\sigma}(\mathbf{r})($ for $\sigma=\alpha$ and $\beta$ ) is the spin-dependent electron density defined by

$$
\rho_{\sigma}(\mathbf{r})=\sum_{i=1}^{N_{\sigma}} w_{i \sigma}\left|\varphi_{i \sigma}(\mathbf{r})\right|^{2} .
$$


Here, $N_{\sigma}$ is the total number of electrons with spin $\sigma$ and $w_{i \sigma}$ is the occupied number of electrons in the spin-orbital $\varphi_{i \sigma}(\mathbf{r})$.

The XC potential can be decomposed into the exchange potential $V_{x \sigma}(\mathbf{r})$ and the correlation potential $V_{c \sigma}(\mathbf{r})$. In the SLHF density-functional approach, the exchange potential is the SLHF exchange potential $V_{x \sigma}^{\mathrm{SLHF}}(\mathbf{r})$ given by

$$
V_{x \sigma}^{\mathrm{SLHF}}(\mathbf{r})=V_{x \sigma}^{\mathrm{S}}(\mathbf{r})+V_{x \sigma}^{\mathrm{C}}(\mathbf{r})
$$

where $V_{x \sigma}^{\mathrm{S}}(\mathbf{r})$ is the Slater potential [10]

$$
V_{x \sigma}^{\mathrm{S}}(\mathbf{r})=-\frac{1}{\rho_{\sigma}(\mathbf{r})} \sum_{i, j=1}^{N_{\sigma}} \varphi_{i \sigma}(\mathbf{r}) \varphi_{j \sigma}(\mathbf{r}) \int \frac{\varphi_{i \sigma}\left(\mathbf{r}^{\prime}\right) \varphi_{j \sigma}\left(\mathbf{r}^{\prime}\right)}{\left|\mathbf{r}-\mathbf{r}^{\prime}\right|} \mathrm{d} \mathbf{r}^{\prime},
$$

and $V_{x \sigma}^{\mathrm{C}}(\mathbf{r})$ is a correction to the Slater potential

$$
V_{x \sigma}^{\mathrm{C}}(\mathbf{r})=\frac{1}{\rho_{\sigma}(\mathbf{r})} \sum_{i, j=1}^{N_{\sigma}} \varphi_{i \sigma}(\mathbf{r}) \varphi_{j \sigma}(\mathbf{r})\left\langle\varphi_{j \sigma}\left|V_{x \sigma}^{\mathrm{SLHF}}-V_{x \sigma}^{\mathrm{NL}}\right| \varphi_{i \sigma}\right\rangle .
$$

Here, $V_{x \sigma}^{\mathrm{NL}}$ is a nonlocal exchange operator of the form of the HF exchange potential but constructed from KS spin-orbitals.

The SLHF exchange potential determined by equations (6)-(8) has two arbitrary additive constants. To settle down the constants so as to pick up the physical orbitals, it is required that the highest-occupied-orbital $N_{\sigma}$ of each spin $\sigma$ does not contribute to the correction term $V_{x \sigma}^{\mathrm{C}}(\mathbf{r})$. In this case, the correction term $V_{x \sigma}^{\mathrm{C}}(\mathbf{r})$ decays exponentially, the SLHF exchange potential behaves asymptotically as the Slater potential and thus approaches to $-1 / r$ at long range [7].

For atomic systems, an electron is characterized by three quantum numbers: the principal quantum number $n$, orbital angular momentum quantum number $l$ and spin $\sigma$. In the spherical coordinates, the spin-orbital $\varphi_{i \sigma}(\mathbf{r})$ of an electron with quantum numbers $n, l$ and $\sigma$ can be expressed by

$$
\varphi_{i \sigma}(\mathbf{r})=\frac{R_{n l \sigma}(r)}{r} Y_{l m}(\theta, \phi),
$$

where $R_{n l \sigma}(r)$ is the radial spin-orbital, $Y_{l m}(\theta, \phi)$ is the spherical harmonic, $m$ is the azimuthal quantum number and $i$ is a set of quantum numbers apart from spin $\sigma$ of the spin-orbital. The radial spin-orbital $R_{n l \sigma}(r)$ is governed by a radial KS equation

$$
\left[-\frac{1}{2} \frac{\mathrm{d}^{2}}{\mathrm{~d} r^{2}}+\frac{l(l+1)}{2 r^{2}}+v_{\sigma}^{\text {eff }}(r)\right] R_{n l \sigma}=\varepsilon_{n l \sigma} R_{n l \sigma},
$$

where $v_{\sigma}^{\text {eff }}(r)$ is the radial effective potential given by

$$
v_{\sigma}^{\mathrm{eff}}(r)=v_{\mathrm{ext}}(r)+v_{H}(r)+v_{x \sigma}^{\mathrm{SLHF}}(r)+v_{c \sigma}(r) .
$$

In equation (11), $v_{\text {ext }}(r), v_{H}(r), v_{x \sigma}^{\mathrm{SLHF}}(r)$ and $v_{c \sigma}(r)$ are the radial external potential, radial Hartree potential, radial SLHF exchange potential and radial correlation potential, respectively.

For an atomic system with a nuclear charge $Z$, the external potential is the Coulomb potential between electron and nucleus

$$
v_{\text {ext }}(r)=-\frac{Z}{r} \text {. }
$$

In the central-field approach, the radial Hartree potential is calculated from

$$
v_{H}(r)=4 \pi \int \frac{\rho\left(r^{\prime}\right)}{r_{>}} r^{\prime 2} \mathrm{~d} r^{\prime},
$$


where $r_{>}$is the larger of $r$ and $r^{\prime}, \rho(r)=\rho_{\alpha}(r)+\rho_{\beta}(r)$ is the spherically averaged total electron density and $\rho_{\sigma}(r)(\sigma=\alpha$ or $\beta)$ is the spherically averaged spin-dependent electron density given by

$$
\rho_{\sigma}(r)=\frac{1}{4 \pi} \int \rho_{\sigma}(\mathbf{r}) \mathrm{d} \Omega=\frac{1}{4 \pi} \sum_{n l}^{v_{\sigma}} w_{n l \sigma}\left[\frac{R_{n l \sigma}}{r}\right]^{2} .
$$

Here, the symbol $v_{\sigma}$ stands for a set of quantum numbers for summation and the sum is performed over all the occupied spin-orbitals with spin $\sigma$. This expression is accurate for spherically symmetric (close-shell) states, but it is only an approximation for non-spherically symmetric (open-shell) states. It may induce an error when it is used to evaluate the energy of a non-spherically symmetric state. However, as will be shown, the error is negligible compared to the order of calculated multiplet splitting [14].

The radial SLHF exchange potential is given by

$$
v_{x \sigma}^{\mathrm{SLHF}}(r)=v_{x \sigma}^{\mathrm{S}}(r)+v_{x \sigma}^{\mathrm{C}}(r),
$$

where $v_{x \sigma}^{\mathrm{S}}(r)$ is the radial Slater potential

$$
v_{x \sigma}^{\mathrm{S}}(r)=-\frac{1}{4 \pi \rho_{\sigma}(r)} \sum_{n l m}^{v_{\sigma}} \sum_{n^{\prime} l^{\prime} m^{\prime}}^{v_{\sigma}} s_{n l m, n^{\prime} l^{\prime} m^{\prime}}^{\sigma}(r),
$$

and $v_{x \sigma}^{\mathrm{C}}(r)$ is a correction to the radial Slater potential

$$
v_{x \sigma}^{\mathrm{C}}(r)=\frac{1}{4 \pi \rho_{\sigma}(r)} \sum_{n l m}^{v_{\sigma}} \sum_{n^{\prime} l^{\prime} m^{\prime}}^{v_{\sigma}} c_{n l m, n^{\prime} l^{\prime} m^{\prime}}^{\sigma}(r) .
$$

The matrix elements $s_{n l m, n^{\prime} l^{\prime} m^{\prime}}^{\sigma}(r)$ and $c_{n l m, n^{\prime} l^{\prime} m^{\prime}}^{\sigma}(r)$ in equations (16) and (17), respectively, are given in [9].

To calculate electron spin-orbitals, the Legendre generalized pseudospectral (LGPS) method [15] is used to discretize the radial KS equation (10). This method associated with an appropriate mapping technique can overcome difficulties due to singularity at $r=0$ and long-tail at large $r$ of the Coulomb interaction and thus provides a very effective and efficient numerical algorithm for high-precision solution of the KS equation. Using the electron spinorbitals of an electronic configuration, a single Slater determinant for a specific electronic state is constructed and its total energy is calculated. The total energy is a sum of non-interacting kinetic energy $E_{k}$, external-field energy $E_{\text {ext }}$, Hartree energy $E_{H}$, exchange energy $E_{x}$ and correlation energy $E_{c}$. The values of $E_{k}, E_{\text {ext }}, E_{H}$ and $E_{x}$ are evaluated by

$$
\begin{aligned}
& E_{k}=\sum_{\sigma=\alpha}^{\beta} \sum_{n l}^{v_{\sigma}} w_{n l \sigma} \int R_{n l \sigma}(r)\left(-\frac{1}{2} \frac{\mathrm{d}^{2}}{\mathrm{~d} r^{2}}+\frac{l(l+1)}{2 r^{2}}\right) R_{n l \sigma}(r) \mathrm{d} r, \\
& E_{\mathrm{ext}}=4 \pi \int v_{\mathrm{ext}}(r) \rho(r) r^{2} \mathrm{~d} r, \\
& E_{H}=\frac{1}{2} \sum_{\Pi} \eta_{l m, l^{\prime} m^{\prime}}^{k} F_{n l \sigma, n^{\prime} l^{\prime} \sigma^{\prime}}^{k}
\end{aligned}
$$

and

$$
E_{x}=-\frac{1}{2} \sum_{\Pi} \lambda_{l m, l^{\prime} m^{\prime}}^{k} G_{n l \sigma, n^{\prime} l^{\prime} \sigma^{\prime}}^{k} \delta_{\sigma \sigma^{\prime}},
$$

where $\Pi$ represents a collection of all the quantum numbers involved, the matrix elements $\eta_{l m, l^{\prime} m^{\prime}}^{k}, F_{n l \sigma, n^{\prime} l^{\prime} \sigma^{\prime}}^{k}, \lambda_{l m, l^{\prime} m^{\prime}}^{k}$ and $G_{n l \sigma, n^{\prime} l^{\prime} \sigma^{\prime}}^{k}$ are given in [9]. 
Table 1. Total energies $(E$ (a.u.)) and excitation energies $(\Delta E(\mathrm{eV}))$ of inner-shell excited states $1 \mathrm{~s} 2 \mathrm{~s} n \mathrm{~s}^{2,4} \mathrm{~S}(n=2-8)$ of Li. The ground-state energies obtained from calculations with the PW and LYP correlation potentials and energy functionals are -7.4837 (a.u.) and -7.4872 (a.u.), respectively. Here, 1 a.u. $=27.2116 \mathrm{eV}$ is used.

\begin{tabular}{|c|c|c|c|c|c|c|c|c|c|c|c|c|c|}
\hline \multirow[b]{3}{*}{ States } & \multicolumn{5}{|c|}{$-E$} & \multicolumn{8}{|c|}{$\Delta E$} \\
\hline & \multicolumn{2}{|c|}{ Present work } & \multicolumn{3}{|c|}{ Other theory } & \multicolumn{2}{|c|}{ Present work } & \multicolumn{3}{|c|}{ Other theory } & \multicolumn{3}{|c|}{ Exp. } \\
\hline & $\mathrm{PW}^{a}$ & $\mathrm{LYP}^{b}$ & $\mathrm{WF}^{c}$ & $\mathrm{SPCR}^{d}$ & $\mathrm{ECI}^{e}$ & $\mathrm{PW}^{a}$ & $\mathrm{LYP}^{b}$ & $\mathrm{WF}^{c}$ & $\mathrm{ECI}^{e}$ & $\mathrm{RRV}^{f}$ & $\mathrm{ZBS}^{g}$ & $\mathrm{RPR}^{h}$ & $\mathrm{RBB}^{i}$ \\
\hline $1 \mathrm{~s} 2 \mathrm{~s}^{22} \mathrm{~S}$ & 5.3940 & 5.3822 & 5.3994 & 5.4052 & & 56.8641 & 57.2796 & 57.055 & & & 56.352 & 56.395 & 56.362 \\
\hline $1 \mathrm{~s} 2 \mathrm{~s} 3 \mathrm{~s}^{4} \mathrm{~S}$ & 5.2225 & 5.2451 & & & 5.2127 & 61.5317 & 61.0114 & & 61.637 & 61.69 & & 62.00 & \\
\hline${ }^{2} S$ & $5.2019^{\star}$ & $5.1466^{\star}$ & & & & $62.0917^{\star}$ & $63.6907^{\star}$ & & & & 61.995 & & $62.012^{\star}$ \\
\hline & $5.1475^{\dagger}$ & $5.1418^{\dagger}$ & & & & $63.5728^{\dagger}$ & $63.8310^{\dagger}$ & & & & & & $63.192^{\dagger}$ \\
\hline $1 \mathrm{~s} 2 \mathrm{~s} 4 \mathrm{~s}^{4} \mathrm{~S}$ & 5.1703 & 5.1918 & & & & 62.9513 & 62.4615 & & 63.113 & 63.18 & & & \\
\hline${ }^{2} \mathrm{~S}$ & 5.1288 & 5.1022 & & & & 64.0806 & 64.8999 & & & & 63.17 & 63.16 & 63.292 \\
\hline $1 \mathrm{~s} 2 \mathrm{~s} 5 \mathrm{~s}^{4} \mathrm{~S}$ & 5.1508 & 5.1715 & & & & 63.4819 & 63.0142 & & & 63.73 & & & \\
\hline${ }^{2} S$ & 5.1108 & 5.0855 & & & & 64.5704 & 65.3549 & & & & & & 63.792 \\
\hline $1 \mathrm{~s} 2 \mathrm{~s} 6 \mathrm{~s}^{4} \mathrm{~S}$ & 5.1412 & 5.1616 & & & & 63.7432 & 63.2847 & & & 63.99 & & & \\
\hline${ }^{2} S$ & 5.1018 & 5.0772 & & & & 64.8153 & 65.5810 & & & & & & \\
\hline $1 \mathrm{~s} 2 \mathrm{~s} 7 \mathrm{~s}^{4} \mathrm{~S}$ & 5.1358 & 5.1560 & & & & 63.8901 & 63.4370 & & & 64.33 & & & \\
\hline${ }^{2} S$ & 5.0966 & 5.0724 & & & & 64.9568 & 65.7100 & & & & & & \\
\hline $1 \mathrm{~s} 2 \mathrm{~s} 8 \mathrm{~s}^{4} \mathrm{~S}$ & 5.1324 & 5.1525 & & & & 63.9826 & 63.5315 & & & & & & \\
\hline${ }^{2} S$ & 5.0933 & 5.0695 & & & & 65.0466 & 65.7909 & & & & & & \\
\hline
\end{tabular}

${ }^{a} \mathrm{PW}$ results, ${ }^{b} \mathrm{LYP}$ results, ${ }^{c}[27],{ }^{d}[28],{ }^{e}[34,35],{ }^{f}[40],{ }^{g}[20],{ }^{h}[22],{ }^{i}[25]$. Here, ${ }^{\star}$ for $1 \mathrm{~s} 2 \mathrm{~s}\left({ }^{3} \mathrm{~S}\right) 3 \mathrm{~s}^{2} \mathrm{~S}$ and ${ }^{\dagger}$ for $1 \mathrm{~s} 2 \mathrm{~s}\left({ }^{1} \mathrm{~S}\right) 3 \mathrm{~s}^{2} \mathrm{~S}$.

For a multiplet state that can be completely described by a single Slater determinant, the energy is calculated directly from the single Slater determinant. For a multiplet state that cannot be represented by a single determinant, the energy can be calculated by means of Slater's diagonal sum rule [10] from the single-Slater-determinant energies [9, 11]. Similar procedures have been employed in recent excited-state calculations [16, 17].

\section{Results and discussion}

The procedure described in the preceding section has been applied to the inner-shell excitedstate calculation of open-shell atomic systems: $\mathrm{Li}, \mathrm{B}, \mathrm{Ne}^{+}, \mathrm{Ne}^{2+}, \mathrm{Ne}^{3+}$ and $\mathrm{Na}$. In the calculations, the correlation potential $v_{c \sigma}(r)$ and the energy functional $E_{c}$ are taken to be those of PW's [12] or LYP's [13]. The results obtained with these two correlation potentials and energy functionals are listed in columns PW and LYP in the following tables, respectively.

\subsection{Inner-shell excitation of $\mathrm{Li}$}

Inner-shell excitation of $\mathrm{Li}$ has been the subject of extensive experimental and theoretical studies. Both experimental data [18-26] and theoretical data [27-40] are abundant. In tables 1-3, we present the total and excitation energies of our calculations for inner-shell excited states $1 \mathrm{~s} 2 \mathrm{~s} n \mathrm{~s}^{2,4} \mathrm{~S}(n=2-8), 1 \mathrm{~s} 2 \mathrm{~s} n \mathrm{p}^{2,4} \mathrm{P}(n=2-8)$ and $1 \mathrm{~s} 2 \mathrm{p}^{2}{ }^{2} \mathrm{~S},{ }^{2,4} \mathrm{P}$ and ${ }^{2} \mathrm{D}$, respectively. For comparison, we also list in these tables the representative experimental data (Exp.) $[20,22,25]$ as well as the theoretical results of the density work-functional formalism (WF) [27], saddle-point complex-rotation (SPCR) [28-31], extensive configuration-interaction calculation (ECI) [34-37] and Rayleigh-Ritz variational method (RRV) [40].

For the total energies of inner-shell excited states $1 \mathrm{~s} 2 \mathrm{~s} n \mathrm{~s}^{2,4} \mathrm{~S}(n=2-8)$ given in table 1 , the maximum relative discrepancies of our PW and LYP results are $0.10 \%$ and $0.32 \%$ to the WF results, $0.21 \%$ and $0.43 \%$ to the SPCR results and $0.19 \%$ and $0.62 \%$ to the ECI results, 
Table 2. Total energies ( $E$ (a.u.)) and excitation energies $(\Delta E(\mathrm{eV}))$ of inner-shell excited states $1 \mathrm{~s} 2 \mathrm{~s} n \mathrm{p}^{2,4} \mathrm{P}(n=2-8)$ of $\mathrm{Li}$.

\begin{tabular}{|c|c|c|c|c|c|c|c|c|c|c|c|c|c|}
\hline \multirow[b]{3}{*}{ States } & \multicolumn{5}{|c|}{$-E$} & \multicolumn{8}{|c|}{$\Delta E$} \\
\hline & \multicolumn{2}{|c|}{ Present work } & \multicolumn{3}{|c|}{ Other theory } & \multicolumn{2}{|c|}{ Present work } & \multicolumn{3}{|c|}{ Other theory } & \multicolumn{3}{|c|}{ Exp. } \\
\hline & $\mathrm{PW}^{a}$ & $\mathrm{LYP}^{b}$ & $\mathrm{WF}^{c}$ & $\mathrm{SPCR}^{d}$ & $\mathrm{ECI}^{e}$ & $\mathrm{PW}^{a}$ & $\mathrm{LYP}^{b}$ & $\mathrm{WF}^{c}$ & $\mathrm{ECI}^{e}$ & $\mathrm{RRV}^{f}$ & $\mathrm{ZBS}^{g}$ & $\mathrm{RPR}^{h}$ & $\mathrm{RBB}^{i}$ \\
\hline \multirow{3}{*}{$\begin{array}{r}1 \mathrm{~s} 2 \mathrm{~s} 2 \mathrm{p}{ }^{4} \mathrm{P} \\
{ }^{2} \mathrm{P}\end{array}$} & 5.3811 & 5.4117 & 5.4114 & 5.3678 & \multirow{3}{*}{5.3679} & 57.2151 & 56.4777 & 56.728 & \multirow{3}{*}{57.413} & \multirow[t]{3}{*}{57.47} & 57.385 & \multirow{4}{*}{$\begin{array}{l}57.41 \\
58.912^{\star} \\
60.405^{\dagger}\end{array}$} & \multirow{3}{*}{$\begin{array}{l}57.432 \\
58.912^{\star} \\
60.402^{\dagger}\end{array}$} \\
\hline & $5.3177^{*}$ & $5.2854^{*}$ & \multirow[t]{2}{*}{5.2912} & $5.3133^{\star}$ & & $58.9403^{*}$ & $59.9145^{*}$ & \multirow[t]{2}{*}{59.999} & & & \multirow{3}{*}{$\begin{array}{l}58.912^{\star} \\
60.397^{\dagger}\end{array}$} & & \\
\hline & $5.2407^{\dagger}$ & $5.2223^{\dagger}$ & & $5.2585^{\dagger}$ & & $61.0356^{\dagger}$ & $61.6316^{\dagger}$ & & & & & & \\
\hline $1 \mathrm{~s} 2 \mathrm{~s} 3 \mathrm{p}^{4} \mathrm{P}$ & 5.1994 & 5.2212 & 5.2205 & & 5.1873 & 62.1595 & 61.6610 & 61.923 & 61.262 & 61.33 & & & \\
\hline${ }^{2} \mathrm{P}$ & 5.1589 & 5.1308 & 5.1767 & 5.1841 & & 63.2615 & 64.1220 & 63.115 & & & 62.425 & 62.421 & 62.462 \\
\hline $1 \mathrm{~s} 2 \mathrm{~s} 4 \mathrm{p}^{4} \mathrm{P}$ & 5.1623 & 5.1835 & & & & 63.1690 & 62.6874 & & & & & & \\
\hline${ }^{2} \mathrm{P}$ & 5.1232 & 5.0973 & & & & 64.2330 & 65.0330 & & & & & & \\
\hline $1 \mathrm{~s} 2 \mathrm{~s} 5 \mathrm{p}^{4} \mathrm{P}$ & 5.1470 & 5.1676 & & & & 63.5853 & 63.1200 & & & & & & \\
\hline${ }^{2} \mathrm{P}$ & 5.1081 & 5.0832 & & & & 64.6439 & 65.4167 & & & & & & \\
\hline $1 \mathrm{~s} 2 \mathrm{~s} 6 \mathrm{p}^{4} \mathrm{P}$ & 5.1391 & 5.1594 & & & & 63.8003 & 63.3432 & & & & & & \\
\hline${ }^{2} \mathrm{P}$ & 5.1003 & 5.0759 & & & & 64.8561 & 65.6153 & & & & & & \\
\hline $1 \mathrm{~s} 2 \mathrm{~s} 7 \mathrm{p}^{4} \mathrm{P}$ & 5.1345 & 5.1547 & & & & 63.9255 & 63.4711 & & & & & & \\
\hline${ }^{2} \mathrm{P}$ & 5.0956 & 5.0717 & & & & 64.9840 & 65.7296 & & & & & & \\
\hline $1 \mathrm{~s} 2 \mathrm{~s} 8 \mathrm{p}^{4} \mathrm{P}$ & 5.1315 & 5.1516 & & & & 64.0071 & 63.5554 & & & & & & \\
\hline${ }^{2} \mathrm{P}$ & 5.0928 & 5.0690 & & & & 65.0602 & 65.8031 & & & & & & \\
\hline
\end{tabular}

${ }^{a} \mathrm{PW}$ results, ${ }^{b}$ LYP results, ${ }^{c}[27],{ }^{d}[31,30],{ }^{e}[34,35,37],{ }^{f}[40],{ }^{g}[20],{ }^{h}[22]$ and ${ }^{i}[25]$. Here, ${ }^{\star}$ for $1 \mathrm{~s}\left(2 \mathrm{~s} 2 \mathrm{p}^{3} \mathrm{P}\right){ }^{2} \mathrm{P}$ and ${ }^{\dagger}$ for $1 \mathrm{~s}\left(2 \mathrm{~s} 2 \mathrm{p}^{1} \mathrm{P}\right){ }^{2} \mathrm{P}$.

Table 3. Total energies $(E$ (a.u. $))$ and excitation energies $(\Delta E(\mathrm{eV}))$ of inner-shell doubly excited states $1 \mathrm{~s} 2 \mathrm{p}^{2}{ }^{2} \mathrm{~S},{ }^{2,4} \mathrm{P}$ and ${ }^{2} \mathrm{D}$ of Li.

\begin{tabular}{|c|c|c|c|c|c|c|c|c|c|c|c|c|c|}
\hline \multirow[b]{3}{*}{ States } & \multicolumn{5}{|c|}{$-E$} & \multicolumn{8}{|c|}{$\Delta E$} \\
\hline & \multicolumn{2}{|c|}{ Present work } & \multicolumn{3}{|c|}{ Other theory } & \multicolumn{2}{|c|}{ Present work } & \multicolumn{3}{|c|}{ Other theory } & \multicolumn{3}{|c|}{ Exp. } \\
\hline & $\mathrm{PW}^{a}$ & $\mathrm{LYP}^{b}$ & $\mathrm{WF}^{c}$ & $\mathrm{SPCR}^{d}$ & $\mathrm{ECI}^{e}$ & $\mathrm{PW}^{a}$ & $\mathrm{LYP}^{b}$ & $\mathrm{WF}^{c}$ & $\mathrm{ECI}^{e}$ & $\operatorname{RRV}^{f}$ & $\mathrm{ZBS}^{g}$ & $\mathrm{RPR}^{h}$ & $\mathrm{RBB}^{i}$ \\
\hline $1 \mathrm{~s} 2 \mathrm{p}^{24} \mathrm{P}$ & 5.2526 & 5.2862 & 5.2860 & 5.2453 & 5.2453 & 60.7118 & 59.8927 & 60.140 & 60.750 & 60.74 & & 60.75 & 60.802 \\
\hline${ }^{2} \mathrm{D}$ & 5.2254 & 5.2232 & 5.2356 & 5.2342 & & 61.4520 & 61.6071 & 61.512 & & & 61.065 & 61.065 & 61.062 \\
\hline${ }^{2} \mathrm{P}$ & 5.2382 & 5.1930 & 5.2323 & 5.2137 & 5.2137 & 61.1036 & 62.4289 & 61.602 & & & & & \\
\hline${ }^{2} \mathrm{~S}$ & 5.1761 & 5.1739 & 5.1780 & & & 62.7935 & 62.9486 & 63.079 & & & & & 63.492 \\
\hline
\end{tabular}

${ }^{a}$ PW results, ${ }^{b}$ LYP results, ${ }^{c}[27],{ }^{d}[29,31],{ }^{e}[34-36],{ }^{f}[40],{ }^{g}[20],{ }^{h}[22]$ and ${ }^{i}[25]$.

respectively. It is shown that both the PW and LYP results are in good agreement with those of other complicated $a b$ initio methods and the PW results are a little bit better than the LYP results. For the excitation energy of excited state $1 \mathrm{~s} 2 \mathrm{~s}^{2}{ }^{2} \mathrm{~S}$, the relative deviations of our PW and LYP results to the experimental results are $0.91 \%$ and $1.65 \%$, respectively, while the relative deviation of the WF results to the experimental results is $1.25 \%$. This demonstrates that the PW result is better than both the LYP and WF results. For the excitation energies of excited states $1 \mathrm{~s} 2 \mathrm{~s} n \mathrm{~s}^{2} \mathrm{~S}$ with $n \geqslant 3$, the maximum relative discrepancies of our PW and LYP results to the experimental results are $1.45 \%$ and $2.75 \%$, respectively. For these states, our results have a little bit larger deviations but still agree with the experimental results within the DFT. For the excitation energy of excited state $1 \mathrm{~s} 2 \mathrm{~s} 3 \mathrm{~s}{ }^{4} \mathrm{~S}$, the relative deviations of our PW and LYP results to the experimental result are $0.75 \%$ and $1.60 \%$, respectively, while the relative discrepancies of the ECI and RRV results to the experimental result are $0.59 \%$ and $0.50 \%$, respectively. Hence our PW result is in good agreement with both the experimental result and other theoretical results for this state. For the excitation energies of excited states $1 \mathrm{~s} 2 \mathrm{~s} n \mathrm{~s}{ }^{4} \mathrm{~S}$ with $n>3$, the maximum relative deviations of our PW and LYP results to the RRV results are $0.68 \%$ and $1.39 \%$, respectively. Our PW results are close to the RRV results. Note that for all the excitation energies considered here the PW results are better than the LYP results. As has already been demonstrated $[9,13]$, due to an overestimation of the LYP energy functional 
to correlation energies of atomic systems with smaller $Z$, the LYP results are generally worse than the PW results for atomic systems with $Z \leqslant 8$.

For the total energies of excited states $1 \mathrm{~s} 2 \mathrm{~s} n \mathrm{p}^{2,4} \mathrm{P}(n=2-8)$ given in table 2 , the maximum relative discrepancies of our PW and LYP results are $0.56 \%$ and $0.89 \%$ to the WF results, $0.64 \%$ and $1.03 \%$ to the SPCR results and $0.25 \%$ and $0.82 \%$ to the ECI results, respectively. It is shown again that both the PW and LYP results are in good agreement with those of other complicated $a b$ initio methods. For the excitation energies, the maximum relative deviations of our PW and LYP results to the experimental results are $1.35 \%$ and $2.73 \%$, respectively, while the maximum relative discrepancies of the WF, ECI and RRV results to the experimental results are $1.85 \%, 0.03 \%$ and $0.07 \%$, respectively. Thus, our PW results are better than the WF results.

For the total energies of inner-shell doubly excited states $1 \mathrm{~s} 2 \mathrm{p}^{2}{ }^{2} \mathrm{~S},{ }^{2,4} \mathrm{P}$ and ${ }^{2} \mathrm{D}$ given in table 3, the maximum relative deviations of our PW and LYP results are $0.63 \%$ and $0.75 \%$ to the WF results, $0.47 \%$ and $0.78 \%$ to both the SPCR and ECI results, respectively. This indicates that our PW and LYP results agree well with those of other $a b$ initio methods. For the excitation energies, the relative discrepancies of our PW and LYP results to the experimental result are not more than $1.10 \%$ and $1.50 \%$, respectively, while the maximum relative discrepancies of the WF results to the experimental results are $1.09 \%$. Hence our PW results are close to the WF results.

\subsection{Auger transition of $B$}

The second open-shell atom we have computed is B. In table 4, we present transition energies for Auger transitions from inner-shell excited states $1 \mathrm{~s} 2 \mathrm{~s}^{2} 2 \mathrm{p}^{2}{ }^{2} \mathrm{~S},{ }^{2,4} \mathrm{P}$ and ${ }^{2} \mathrm{D}$ of $\mathrm{B}$ to singly excited states $1 \mathrm{~s}^{2} 2 \mathrm{~s} 2 \mathrm{p}^{1,3} \mathrm{P}$ and doubly excited states $1 \mathrm{~s}^{2} 2 \mathrm{p}^{2}{ }^{1} \mathrm{~S},{ }^{3} \mathrm{P}$ and ${ }^{1} \mathrm{D}$ of $\mathrm{B}^{+}$. For comparison, we also give in this table the available experimental results (Exp.) [25] as well as theoretical results of WF [27], perturbation Z-expansion theory (PZE) [41, 42] and perturbation Z-expansion theory with relativistic effects (RPZE) [25].

It is shown that except for the transitions $1 \mathrm{~s} 2 \mathrm{~s}^{2} 2 \mathrm{p}^{2}{ }^{2} \mathrm{D} \rightarrow 1 \mathrm{~s}^{2} 2 \mathrm{p}^{21} \mathrm{~S}$ and $1 \mathrm{~s} 2 \mathrm{~s}^{2} 2 \mathrm{p}^{2}{ }^{2} \mathrm{~S} \rightarrow$ $1 \mathrm{~s}^{2} 2 \mathrm{p}^{21} \mathrm{~S}$, the maximum deviations of our PW and LYP results to the experimental results are $0.63 \%$ and $0.24 \%$, respectively. Thus, our results are in good agreement with the experimental results. Furthermore, the maximum discrepancies of the WF, PZE and RPZE results to the experimental results are $0.78 \%, 1.21 \%$ and $0.41 \%$, respectively. This indicates that our results are much better than the PZE results, a little bit better than the WF results, and much closer to the RPZE results. As for the transitions $1 \mathrm{~s} 2 \mathrm{~s}^{2} 2 \mathrm{p}^{2}{ }^{2} \mathrm{D} \rightarrow 1 \mathrm{~s}^{2} 2 \mathrm{p}^{21} \mathrm{~S}$ and $1 \mathrm{~s} 2 \mathrm{~s}^{2} 2 \mathrm{p}^{2}{ }^{2} \mathrm{~S} \rightarrow 1 \mathrm{~s}^{2} 2 \mathrm{p}^{21} \mathrm{~S}$, the relative deviations of our PW and LYP results to the experimental results are less than $1.82 \%$ and $1.23 \%$, respectively, while the relative deviations of the PZE and RPZE results to the experimental results are not more than $3.26 \%$ and $0.07 \%$, respectively. This indicates again that our results are much better than the PZE results. In addition, according to our result the spectrum line with $170.7 \mathrm{eV}$ being originally assigned to the transition $1 \mathrm{~s} 2 \mathrm{~s}^{2} 2 \mathrm{p}^{2}{ }^{2} \mathrm{D} \rightarrow 1 \mathrm{~s}^{2} 2 \mathrm{p}^{21} \mathrm{~S}$ may belong to the transition $1 \mathrm{~s} 2 \mathrm{~s}^{2} 2 \mathrm{p}^{2} \mathrm{D} \rightarrow 1 \mathrm{~s}^{2} 2 \mathrm{p}^{23} \mathrm{P}$.

\subsection{Inner-shell excitation of $\mathrm{Ne}^{+}, \mathrm{Ne}^{2+}$ and $\mathrm{Ne}^{3+}$}

To explore the feasibility of the approach to inner-shell excitation of open-shell atomic ions, we also apply the procedure to the inner-shell excited-state calculation of positive ions $\mathrm{Ne}^{+}, \mathrm{Ne}^{2+}$ and $\mathrm{Ne}^{3+}$. In table 5, we present the transition energies of optically allowed transitions involved in inner-shell excited states of these ions along with the theoretical results of multiconfiguration 
Table 4. Transition energies $(\Delta E(\mathrm{eV}))$ for Auger transitions from inner-shell excited states $1 \mathrm{~s} 2 \mathrm{~s}^{2} 2 \mathrm{p}^{2}{ }^{2} \mathrm{~S},{ }^{2,4} \mathrm{P}$ and ${ }^{2} \mathrm{D}$ of $\mathrm{B}$ to singly excited states $1 \mathrm{~s}^{2} 2 \mathrm{~s} 2 \mathrm{p}{ }^{1,3} \mathrm{P}$ and doubly excited states $1 \mathrm{~s}^{2} 2 \mathrm{p}^{2} \mathrm{~S}^{3} \mathrm{P}$ and ${ }^{1} \mathrm{D}$ of $\mathrm{B}^{+}$.

\begin{tabular}{|c|c|c|c|c|c|c|c|}
\hline \multirow[b]{3}{*}{ Initial states } & \multirow[b]{3}{*}{ Final states } & \multicolumn{6}{|c|}{$\Delta E$} \\
\hline & & \multicolumn{2}{|c|}{ Present work } & \multicolumn{3}{|c|}{ Other theory } & \multirow{2}{*}{$\frac{\text { Exp. }}{\mathrm{RBB}^{f}}$} \\
\hline & & $\mathrm{PW}^{a}$ & $\mathrm{LYP}^{b}$ & $\mathrm{WF}^{c}$ & $\mathrm{PZE}^{d}$ & $\mathrm{RPZE}^{e}$ & \\
\hline \multirow[t]{6}{*}{$1 \mathrm{~s} 2 \mathrm{~s}^{2} 2 \mathrm{p}^{2}{ }^{4} \mathrm{P}$} & $1 s^{2} 2 p^{2}{ }^{3} P$ & 168.3500 & 169.1008 & & 167.4101 & 169.10 & 169.2 \\
\hline & $1 \mathrm{~s}^{2} 2 \mathrm{p}^{21} \mathrm{D}$ & 167.3105 & 168.5612 & & 166.7952 & & \\
\hline & $1 s^{2} 2 p^{21} s$ & 165.5064 & 167.2558 & & 163.2454 & & \\
\hline & $1 \mathrm{~s}^{2} 2 \mathrm{p}^{2}{ }^{3} \mathrm{P}$ & 170.4344 & 170.7805 & & 169.3045 & & \\
\hline & $1 \mathrm{~s}^{2} 2 \mathrm{p}^{21} \mathrm{D}$ & 169.3949 & 170.2409 & & 168.6895 & & \\
\hline & $1 s^{2} 2 p^{2}{ }^{1} s$ & 167.5908 & 168.9356 & & 165.1397 & 170.58 & 170.7 \\
\hline \multirow[t]{3}{*}{${ }^{2} \mathrm{P}$} & $1 \mathrm{~s}^{2} 2 \mathrm{p}^{23} \mathrm{P}$ & 170.4181 & 170.2883 & 171.5858 & 169.0290 & 170.73 & 170.7 \\
\hline & $1 \mathrm{~s}^{2} 2 \mathrm{p}^{21} \mathrm{D}$ & 169.3786 & 169.7487 & & 168.4140 & & \\
\hline & $1 s^{2} 2 p^{21} s$ & 167.5745 & 168.4433 & & 164.8643 & & \\
\hline \multirow[t]{3}{*}{${ }^{2} \mathrm{~S}$} & $1 \mathrm{~s}^{2} 2 \mathrm{p}^{23} \mathrm{P}$ & 172.7828 & 173.1289 & & 171.0097 & & \\
\hline & $1 \mathrm{~s}^{2} 2 \mathrm{p}^{21} \mathrm{D}$ & 171.7433 & 172.5893 & & 170.3947 & & \\
\hline & $1 s^{2} 2 p^{2}{ }^{1} S$ & 169.9392 & 171.2840 & & 166.8450 & 169.13 & 169.2 \\
\hline \multirow[t]{4}{*}{$1 s 2 s^{2} 2 p^{2}{ }^{4} P$} & $1 s^{2} 2 s 2 p^{3} P$ & 176.1516 & 176.8376 & & 175.7192 & & \\
\hline & $1 s^{2} 2 s 2 p^{1} \mathrm{P}$ & 171.4249 & 172.9961 & & 170.8674 & & \\
\hline & $1 s^{2} 2 s^{2} p^{3} P$ & 178.2360 & 178.5173 & & 177.6135 & & \\
\hline & $1 \mathrm{~s}^{2} 2 \mathrm{~s} 2 \mathrm{p}^{1} \mathrm{P}$ & 173.5093 & 174.6759 & 173.2321 & 172.7617 & 174.17 & 174.6 \\
\hline \multirow[t]{2}{*}{${ }^{2} \mathrm{P}$} & $1 s^{2} 2 s^{2} p^{3} P$ & 178.2197 & 178.0251 & & 177.3381 & & \\
\hline & $1 \mathrm{~s}^{2} 2 \mathrm{~s} 2 \mathrm{p}^{1} \mathrm{P}$ & 173.4930 & 174.1836 & 173.4062 & 172.4862 & 173.90 & 174.6 \\
\hline \multirow[t]{2}{*}{${ }^{2} \mathrm{~S}$} & $1 s^{2} 2 s 2 p^{3} P$ & 180.5843 & 180.8657 & & 179.3188 & & \\
\hline & $1 s^{2} 2 s^{2} p^{1} P$ & 175.8577 & 177.0242 & & 174.4669 & & \\
\hline
\end{tabular}

${ }^{a} \mathrm{PW}$ results, ${ }^{b}$ LYP results, ${ }^{c}[27],{ }^{d}[41,42],{ }^{e}[25]$ and ${ }^{f}[25]$.

Dirac-Fock method (MCDF) [43]. Note that the MCDF results given in this table are the weighted-averaged values of those in [43].

It is shown that the relative deviations of our PW and LYP results to the MCDF results are not more than $0.17 \%$ and $0.07 \%$ for $\mathrm{Ne}^{+}, 0.16 \%$ and $0.06 \%$ for $\mathrm{Ne}^{2+}$ and $0.16 \%$ and $0.08 \%$ for $\mathrm{Ne}^{3+}$, respectively. The satisfactory agreement of our results with the MCDF results demonstrates that the SLHF together with the PW or LYP correlation potential and energy functional are accurate for the calculation of inner-shell excited states of positive ions. It is also shown that for the atomic systems with $Z=10$ the LYP results are more accurate than the PW results, as has already been demonstrated in our previous work [9, 11].

\subsection{Inner-shell excitation of $\mathrm{Na}$}

Finally, we calculate the total and excitation energies of inner-shell excited states $1 \mathrm{~s} 2 \mathrm{~s}^{2} 2 \mathrm{p}^{6} 3 \mathrm{~s}\left({ }^{1} \mathrm{~S}\right) n \mathrm{p}^{2} \mathrm{P}(n=3-8)$ and $1 \mathrm{~s} 2 \mathrm{~s}^{2} 2 \mathrm{p}^{6} 3 \mathrm{~s}\left({ }^{3} \mathrm{~S}\right) n \mathrm{p}^{2,4} \mathrm{P}(n=3-8)$ of Na. The results are shown in table 6 together with experimental results (Exp.) [44] as well as theoretical results of the relativistic configuration interaction method (RCI) [45] and multiconfiguration Hartree-Fock method (MCHF) [46] for comparison.

For the excitation energies of excited states $1 \mathrm{~s} 2 \mathrm{~s}^{2} 2 \mathrm{p}^{6} 3 \mathrm{~s}\left({ }^{1} \mathrm{~S}\right) n \mathrm{p}^{2} \mathrm{P}$ with $n=3-5$, the relative deviations of our PW and LYP results to the experimental results are less than $0.18 \%$ and $0.07 \%$, respectively, while the maximum relative discrepancies of the RCI and MCHF 
Table 5. Transition energies $(\Delta E(\mathrm{eV}))$ of optically allowed transitions of inner-shell excited states of $\mathrm{Ne}^{+}, \mathrm{Ne}^{2+}$ and $\mathrm{Ne}^{3+}$

\begin{tabular}{|c|c|c|c|c|c|}
\hline \multirow[b]{3}{*}{ Ions } & \multirow[b]{3}{*}{ Initial state } & \multirow[b]{3}{*}{ Final state } & \multicolumn{3}{|c|}{$\Delta E$} \\
\hline & & & \multicolumn{2}{|c|}{ Present work } & \multirow{2}{*}{$\frac{\text { Others }}{\mathrm{MCDF}^{c}}$} \\
\hline & & & $\mathrm{PW}^{a}$ & $\mathrm{LYP}^{b}$ & \\
\hline $\mathrm{Ne}^{+}$ & $1 s^{2} 2 s^{2} 2 p^{52} P$ & $1 s 2 s^{2} 2 p^{62} S$ & 848.0278 & 848.8786 & 849.48 \\
\hline \multirow[t]{3}{*}{$\mathrm{Ne}^{2+}$} & $1 s^{2} 2 s^{2} 2 p^{43} P$ & $1 s 2 s^{2} 2 p^{53} P$ & 853.7724 & 854.6535 & 855.16 \\
\hline & ${ }^{1} \mathrm{D}$ & ${ }^{1} \mathrm{P}$ & 855.0747 & 856.3277 & 856.14 \\
\hline & ${ }^{1} \mathrm{~S}$ & ${ }^{1} \mathrm{P}$ & 850.1188 & 851.3728 & 851.19 \\
\hline \multirow[t]{6}{*}{$\mathrm{Ne}^{3+}$} & $1 s^{2} 2 s^{2} 2 p^{34} s$ & $1 s 2 s^{2} 2 p^{44} P$ & 861.0411 & 861.8325 & 862.41 \\
\hline & ${ }^{2} \mathrm{D}$ & ${ }^{2} \mathrm{D}$ & 862.2075 & 863.0207 & 863.33 \\
\hline & & ${ }^{2} \mathrm{P}$ & 863.1715 & 863.6460 & 864.20 \\
\hline & ${ }^{2} \mathrm{P}$ & ${ }^{2} \mathrm{D}$ & 858.5203 & 859.3295 & 859.78 \\
\hline & & ${ }^{2} \mathrm{P}$ & 859.4843 & 859.9549 & 860.65 \\
\hline & & ${ }^{2} \mathrm{~S}$ & 864.5179 & 865.3302 & 865.62 \\
\hline
\end{tabular}

${ }^{a}$ PW results, ${ }^{b}$ LYP results and ${ }^{c}$ obtained from data in [43].

Table 6. Total energies $(E$ (a.u.)) and excitation energies $(\Delta E(\mathrm{eV}))$ of inner-shell excited states $1 \mathrm{~s} 2 \mathrm{~s}^{2} 2 \mathrm{p}^{6} 3 \mathrm{~s}\left({ }^{1} \mathrm{~S}\right) n \mathrm{p}^{2} \mathrm{P}$ and $1 \mathrm{~s} 2 \mathrm{~s}^{2} 2 \mathrm{p}^{6} 3 \mathrm{~s}\left({ }^{3} \mathrm{~S}\right) n \mathrm{p}^{2,4} \mathrm{P}(n=3-8)$ of Na. The ground-state energies obtained from calculations with the PW and LYP correlation potentials and energy functionals are -162.2265 (a.u.) and -162.2687 (a.u.), respectively.

\begin{tabular}{|c|c|c|c|c|c|c|c|}
\hline \multirow[b]{3}{*}{ States } & \multirow{2}{*}{\multicolumn{2}{|c|}{$\frac{-E}{\text { Present work }}$}} & \multicolumn{5}{|c|}{$\Delta E$} \\
\hline & & & \multicolumn{2}{|c|}{ Present work } & \multicolumn{2}{|c|}{ Other theory } & \multirow{2}{*}{$\frac{\text { Exp. }}{\text { TLE }^{e}}$} \\
\hline & $\mathrm{PW}^{a}$ & $\mathrm{LYP}^{b}$ & $\mathrm{PW}^{a}$ & $\mathrm{LYP}^{b}$ & $\mathrm{RCI}^{c}$ & $\mathrm{MCHF}^{d}$ & \\
\hline $1 s 2 s^{2} 2 p^{6} 3 s\left({ }^{1} s\right) 3 p^{2} P$ & 122.7947 & 122.7552 & 1073.0024 & 1074.1765 & 1074.28 & 1074.50 & 1074.95 \\
\hline $4 p^{2} \mathrm{P}$ & 122.6680 & 122.6708 & 1076.4501 & 1077.5222 & 1077.52 & 1077.96 & 1078.17 \\
\hline $5 \mathrm{p}^{2} \mathrm{P}$ & 122.6378 & 122.6424 & 1077.2719 & 1078.2950 & 1078.27 & 1078.60 & 1078.9 \\
\hline $6 \mathrm{p}^{2} \mathrm{P}$ & 122.6246 & 122.6301 & 1077.6311 & 1078.6297 & & & \\
\hline $7 \mathrm{p}^{2} \mathrm{P}$ & 122.6176 & 122.6234 & 1077.8215 & 1078.8120 & & & \\
\hline $8 \mathrm{p}^{2} \mathrm{P}$ & 122.6134 & 122.6195 & 1077.9358 & 1078.9182 & & & \\
\hline $1 s 2 s^{2} 2 p^{6} 3 s\left({ }^{3} s\right) 3 p^{2} P$ & 122.7540 & 122.7714 & 1074.1099 & 1074.7847 & 1075.97 & 1076.04 & 1076.47 \\
\hline $4 p^{2} \mathrm{P}$ & 122.6858 & 122.6976 & 1075.9657 & 1076.7929 & 1077.21 & 1077.83 & \\
\hline $5 p^{2} \mathrm{P}$ & 122.6601 & 122.6705 & 1076.6651 & 1077.5304 & 1077.88 & 1078.56 & \\
\hline $6 \mathrm{p}^{2} \mathrm{P}$ & 122.6481 & 122.6582 & 1076.9916 & 1077.8651 & & & \\
\hline $7 \mathrm{p}^{2} \mathrm{P}$ & 122.6414 & 122.6517 & 1077.1739 & 1078.0420 & & & \\
\hline $8 \mathrm{p}^{2} \mathrm{P}$ & 122.6374 & 122.6478 & 1077.2828 & 1078.1481 & & & \\
\hline $3 p^{4} P$ & 122.8152 & 122.8176 & 1072.4445 & 1073.5276 & 1073.87 & & \\
\hline $4 p^{4} P$ & 122.6916 & 122.6983 & 1075.8079 & 1076.7739 & 1076.94 & & \\
\hline $5 p^{4} \mathrm{P}$ & 122.6619 & 122.6703 & 1076.6161 & 1077.5358 & 1077.71 & & \\
\hline $6 p^{4} P$ & 122.6487 & 122.6580 & 1076.9753 & 1077.8705 & & & \\
\hline $7 p^{4} P$ & 122.6418 & 122.6515 & 1077.1630 & 1078.0474 & & & \\
\hline $8 p^{4} P$ & 122.6377 & 122.6476 & 1077.2746 & 1078.1535 & & & \\
\hline
\end{tabular}

${ }^{a} \mathrm{PW}$ results, ${ }^{b}$ LYP results, ${ }^{c}[45],{ }^{d}[46]$ and ${ }^{e}[44]$.

results to the experimental results are $0.06 \%$ and $0.04 \%$, respectively. This indicates that both our PW and LYP results are in good agreement with the experimental results, the LYP results are better than the PW results and the LYP results are very close to the RCI and MCHF results. 
It is also shown that according to our results the identification of the photoionization spectra $1 s 2 s^{2} 2 p^{6} 3 s\left({ }^{1} S\right) 3 p^{2} \mathrm{P}$ and $1 s 2 s^{2} 2 p^{6} 3 s\left({ }^{3} S\right) 3 p^{2} \mathrm{P}$ in the experiment [44] should be exchanged. This agrees well with the RCI and MCHF results.

For the excitation energy of excited state $1 s^{2} s^{2} 2 p^{6} 3 s\left({ }^{3} S\right) 3 p^{2} \mathrm{P}$, the relative deviations of our PW and LYP results to the experimental results are $0.22 \%$ and $0.16 \%$, respectively, which are a little bit larger than those of RCI and MCHF results, $0.05 \%$ and $0.04 \%$. For the excitation energies of excited states $1 \mathrm{~s}^{2} \mathrm{~s}^{2} 2 \mathrm{p}^{6} 3 \mathrm{~s}\left({ }^{3} \mathrm{~S}\right) n \mathrm{p}^{2} \mathrm{P}$ with $n=4$ and 5 , the relative deviations of our PW and LYP results are not more than $0.12 \%$ and $0.04 \%$ to the RCI results and $0.18 \%$ and $0.10 \%$ to the MCHF results, respectively. Thus, the agreement of our results with the experimental and other theoretical results is good and our LYP results are closer to the RCI results.

For the excitation energies of excited states $1 \mathrm{~s} 2 \mathrm{~s}^{2} 2 \mathrm{p}^{6} 3 \mathrm{~s}\left({ }^{3} \mathrm{~S}\right) n \mathrm{p}^{4} \mathrm{P}$ with $n=3-5$, the relative deviations of our PW and LYP results to the RCI results are less than $0.13 \%$ and $0.03 \%$, respectively. This shows again that our LYP results are in very good agreement with the RCI results. Note that for all the excited states discussed here the LYP results are better than the PW results. Thus, the LYP correlation potential and energy functional is more accurate than those of PW for the inner-shell excited-state calculation of open-shell atomic systems with large $Z$.

\section{Conclusions}

In summary, the SLHF density-functional approach together with Slater's diagonal sum rule are extended to the inner-shell excited-state calculation of open-shell atomic systems. In this procedure, the electron spin-orbitals in an electronic configuration are obtained first by solving the KS equation with the exact SLHF exchange potential. Then the single-Slaterdeterminant energies of the electronic configuration are calculated by using these electron spin-orbitals. And finally the multiplet energy of an excited state is evaluated from the singleSlater-determinant energies in terms of Slater's diagonal sum rule. This procedure has been used to calculate the total and excitation energies of inner-shell excited states of open-shell atomic systems: $\mathrm{Li}, \mathrm{B}, \mathrm{Ne}^{+}, \mathrm{Ne}^{2+}, \mathrm{Ne}^{3+}$ and $\mathrm{Na}$. The electron correlation effect is taken into account by incorporating the PW or LYP correlation potential and energy functional into the calculation. It is shown that the PW results are better for atomic systems with $Z \leqslant 8$, while the LYP results are better for atomic systems with $Z>8$. They are in good agreement with each other and also with the available experimental as well as other $a b$ initio theoretical results. Therefore, the SLHF density-functional approach provides a powerful and computationally efficient scheme for the accurate calculation of inner-shell excited states of open-shell atomic systems within DFT.

\section{Acknowledgments}

This work is partially supported by the Chemical Sciences, Geosciences and Biosciences Division of the Office of Basic Energy Sciences, Office of Science, US Department of Energy and by the National Science Foundation.

\section{References}

[1] Hohenberg P and Kohn W 1964 Phys. Rev. 136 B864

[2] Kohn W and Sham L J 1965 Phys. Rev. 140 A1133 
[3] Dreizler R M and Gross E K U 1990 Density Functional Theory: An Approach to The Quantum Many-Body Problem (Berlin: Springer)

[4] Parr R G and Yang W 1989 Density-Functional Theory of Atoms and Molecules (New York: Oxford University Press)

[5] Becke A D 1988 Phys. Rev. A 383098

[6] Perdew J P and Wang Y 1986 Phys. Rev. B 338800

[7] Sala F D and Görling A 2001 J. Chem. Phys. 1155718

[8] Sala F D and Görling A 2002 Phys. Rev. Lett. 89033003

[9] Zhou Z and Chu S I 2005 Phys. Rev. A 71022513

[10] Slater J C 1960 Quantum Theory of Atomic Structure vol 2 (New York: McGraw-Hill)

[11] Zhou Z and Chu S I 2007 Phys. Rev. A 75014501

[12] Perdew J P and Wang Y 1992 Phys. Rev. B 4513244

[13] Lee C, Yang W and Parr R G 1988 Phys. Rev. B 37785

[14] Singh R and Deb B M 1999 Phys. Rep. 31147

[15] Wang J, Chu S I and Laughlin C 1994 Phys. Rev. A 503208

[16] Roy A K and Chu S I 2002 Phys. Rev. A 65052508

[17] Pollak C, Rosa A and Baerends E J 1997 J. Am. Chem. Soc. 1197324

[18] Feldman P and Novick R 1967 Phys. Rev. 160143

[19] Ederer D L, Lucatorto T and Madden R P 1970 Phys. Rev. Lett. 251537

[20] Ziem P, Bruch R and Stolterfoht N 1975 J. Phys. B: At. Mol. Phys. 8 L480

[21] Pegg D J, Haselton H H, Thoe R S, Griffin P M, Brown M D and Sellin I A 1975 Phys. Rev. A 121330

[22] Rassi D, Pejčev V and Ross K J 1977 J. Phys. B: At. Mol. Phys. 103535

[23] McIlrath T J and Lucatorto T B 1977 Phys. Rev. Lett. 381390

[24] Cantù A M, Parkinson W H, Tondello G and Tozzi G P 1977 J. Opt. Soc. Am. 671030

[25] Rødbro M, Bruch R and Bisgaard P 1979 J. Phys. B: At. Mol. Phys. 122413

[26] Mannervik H and Cederquist H 1985 Phys. Scr. 3179

[27] Roy A K and Deb B M 1997 Phys. Lett. A 234465

[28] Davis B F and Chung K T 1984 Phys. Rev. A 291878

[29] Davis B F and Chung K T 1988 Phys. Rev. A 37111

[30] Davis B F and Chung K T 1989 Phys. Rev. A 393942

[31] Chen M K and Chung K T 1994 Phys. Rev. A 491675

[32] Wakid S, Bhatia A K and Temkin A 1980 Phys. Rev. A 21496

[33] Bhatia A K and Temkin A 1976 Phys. Rev. A 132322

[34] Bunge C F and Bunge A V 1978 Phys. Rev. A 17816

[35] Bunge C F and Bunge A V 1978 Phys. Rev. A 17822

[36] Bunge C F 1979 Phys. Rev. A 19936

[37] Bunge C F 1981 J. Phys. B: At. Mol. Phys. 141

[38] Lunell S and Beebe N H F 1977 Phys. Scr. 15268

[39] Lunell S 1977 Phys. Scr. 1613

[40] Holøien S and Geltman S 1967 Phys. Rev. 15381

[41] Safronova U I and Kharitonova V N 1969 Opt. Spectrosc. 27300

[42] Ivanova E P and Safronova U I 1975 J. Phys. B: At. Mol. Phys. 81591

[43] Yamaoka H, Oura M, Kawatsura K, Hayaishi T, Sekioka T, Agui A, Yoshigoe A and Koike F 2001 Phys. Rev. A 65012709

[44] Tuilier M H, Laporte D and Esteva J M 1982 Phys. Rev. A 26372

[45] Yang L 1993 J. Phys. B: At. Mol. Opt. Phys. 261813

[46] Yavna V A, Petrov I D, Demekhina L A, Khoperskii A N and Sukhorukov V L 1986 Opt. Spectrosc. 61552 